\title{
Tunable Rashba Spin Splitting in Two-Dimensional Polar Perovskites
}

\author{
Jiajia Chen, ${ }^{1}$ Kai Wu, ${ }^{1}$ Wei Hu, ${ }^{1, *}$ and Jinlong Yang ${ }^{1, \dagger}$ \\ ${ }^{1}$ Department of Chemical Physics, Hefei National Laboratory for Physical Sciences at the Microscale, \\ and Synergetic Innovation Center of Quantum Information and Quantum Physics, \\ University of Science and Technology of China, Hefei, Anhui 230026, China
}

(Dated: February 11, 2021)

\section{ELECTRONIC PROPERTIES OF C-RBPBI}

As shown in Fig. S1, $\mathrm{C}-\mathrm{RbPbI}_{3}$ has the largest band splitting around the $\mathrm{M}$ point among $\mathrm{C}-\mathrm{ABX}_{3}$, but their spin textures around the M point of Rashba bands do not have opposite rotation. Our spin arrows around M point are difficult to see clearly as shown in Fig. S1(c) and (e). So, we add spin textures without the characteristic of positive (negative) spin component in $\mathrm{S} 1$ (d) and (f), in order to show the spin of M more clearly. Similar to C-RbPbI 3 , other $\mathrm{C}-\mathrm{ABX}_{3}$ have similar but smaller band splitting. Thus, C-ABX 3 lack Rashba effect.
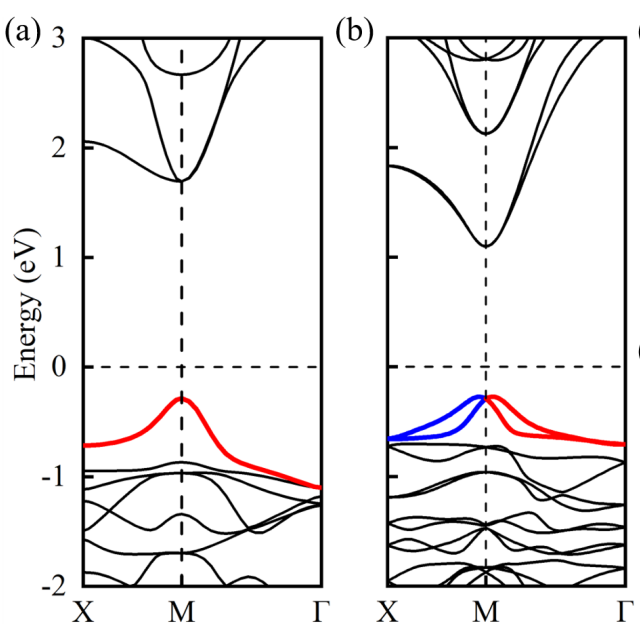
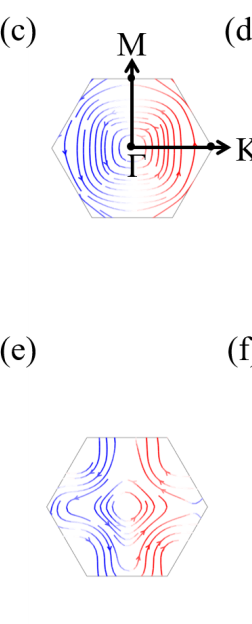

(d)

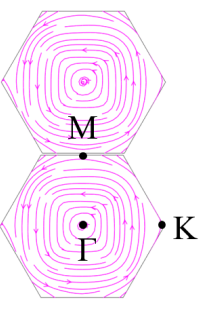

(f)

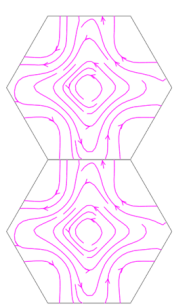

Fig. S1: Electonic structures of C-RbPbI $\mathrm{I}_{3}$ Band structures of C-RbPbI 3 by (a) PBE and (b) PBE+SOC. Characteristic spin textures of (c) upper and (e) lower the Rashba bands of VB. Red (blue) represents positive (negative) spin component along y-axis, respectively. Characteristic spin textures of (d) upper and (f) lower the Rashba bands of $\mathrm{VB}$, where color does not represent physical properties.

\footnotetext{
*E-mail: whuustc@ustc.edu.cn (Wei Hu)

†E-mail: jlyang@ustc.edu.cn (Jinlong Yang)
} 

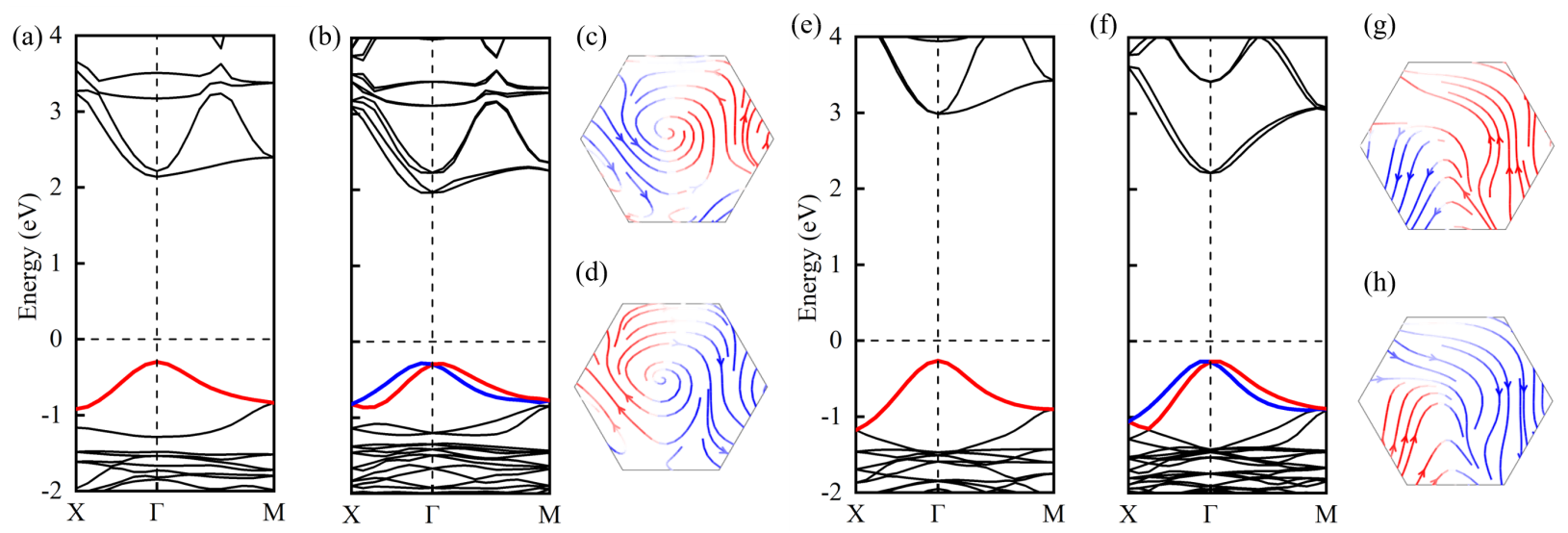

Fig. S2: Electronic structures of $2 \mathrm{D}$ polar perovskites by hybrid calculations. Band structures of $\mathrm{O}-\mathrm{RbSnI} 3 \mathrm{by}(\mathrm{a})$ HSE06 and (b) HSE06+SOC. Characteristic spin textures of (c) upper and (d) lower Rashba bands of VB. Band structures of T-RbPbBr 3 by (e) HSE06 and (f) HSE06+SOC. Characteristic spin textures of (g) upper and (h) lower Rashba bands of VB. The horizontal dashed lines indicate the Fermi level. 


\section{SPIN PROPERTIES OF CBM BANDS OF O-RBSNI AND T-RBPBBR $_{3}$}

Although 2D polar perovskites have band splitting at the CBM bands, corresponding spin textures do not have opposite directions as shown in Fig. S3. 3D images of CBM bands are more vivid, which are also shown in Fig. S3.

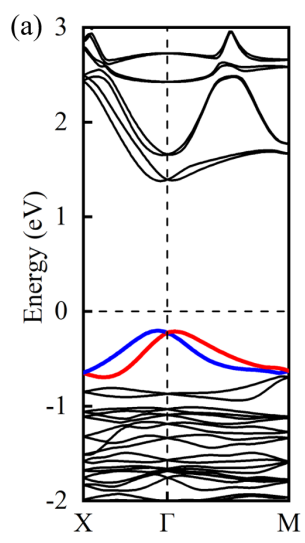

(b)

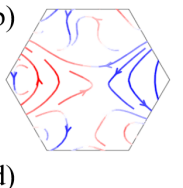

(d)

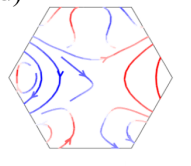

(e)

(c)
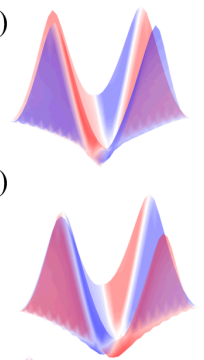

(f)

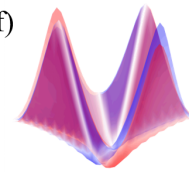

(g)

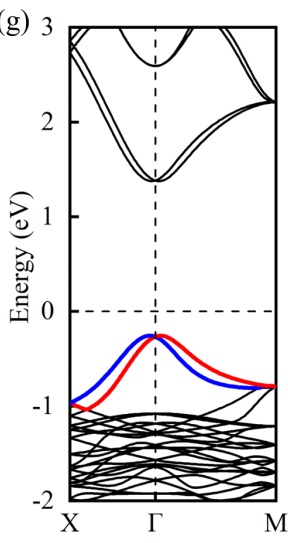

(h)

(h)

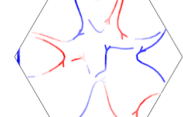

(j)

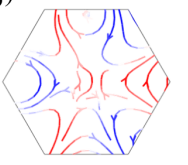

is

k)
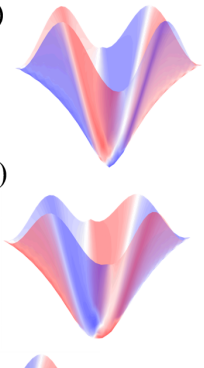

(1)

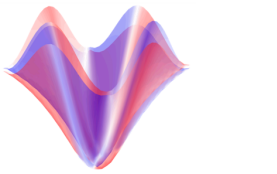

Fig. S3: (a) Band structures of O-RbSnI 3 by PBE+SOC. (b) Characteristic spin textures and 3D image (c) of upper CBM band. (d) Characteristic spin textures and 3D image (e) of lower CBM band. (f) 3D image of CBM bands. (g) Band structures of $\mathrm{T}-\mathrm{RbPbBr}_{3}$ by $\mathrm{PBE}+\mathrm{SOC}$. (h) Characteristic spin textures and 3D image (i) of upper CBM band. (j) Characteristic spin textures and 3D image (k) of lower CBM band. (l) 3D image of CBM bands. Red (blue) represents positive (negative) spin component along y-axis, respectively. 


\section{MECHANISM ANALYSIS}

The net dipole is the most relevant to the strength of intrinsic Rashba splitting. We list the dipole moment of $\mathrm{T}_{-} \mathrm{ABX}_{3}$ and $\mathrm{O}-\mathrm{ABX}_{3}$ in Table $\mathrm{S} 1$. These perovskites are $2 \mathrm{D}$ materials rather than bulk materials. Therefore, the net dipole and induced Rashba filed are indeed in-plane.

Table S1: Dipole moment $\mu(\mathrm{e} \cdot \AA)$ of $2 \mathrm{D}$ polar perovskites $\mathrm{T}-\mathrm{ABX}_{3}$ and $\mathrm{O}-\mathrm{ABX}_{3}$.

\begin{tabular}{ccc}
\hline \hline & $\mathrm{T}-\mathrm{ABX}$ & $\mathrm{O}-\mathrm{ABX}$ \\
\hline & -0.280 & -0.282 \\
$\mathrm{CsPbCl}_{3}$ & -0.350 & -0.339 \\
$\mathrm{CsPbBr}_{3}$ & -0.405 & -0.383 \\
$\mathrm{CsPbI}_{3}$ & -0.461 & -0.360 \\
$\mathrm{CsSnCl}_{3}$ & -0.473 & -0.422 \\
$\mathrm{CsSnBr}_{3}$ & -0.474 & -0.461 \\
$\mathrm{CsSnI}_{3}$ & -0.128 & -0.131 \\
$\mathrm{RbPbCl}_{3}$ & -0.207 & -0.174 \\
$\mathrm{RbPbBr}_{3}$ & -0.277 & -0.240 \\
$\mathrm{RbPbI}_{3}$ & -0.325 & -0.199 \\
$\mathrm{RbSnCl}_{3}$ & -0.350 & -0.228 \\
$\mathrm{RbSnBr}_{3}$ & -0.365 & -0.259 \\
$\mathrm{RbSnI}_{3}$ &
\end{tabular}

Table S2: Electrostatic potential energy and Bader charge analysis of $2 \mathrm{D} \mathrm{ABX}_{3}$ perovskites. $\Delta \phi(\mathrm{eV})$ is the workfunction difference between $\mathrm{BX}_{2}$ and $\mathrm{AX}$ side. $\mathrm{Q}_{\mathrm{B}}$ is the average charge of $\mathrm{B}$ site $(\mathrm{Pb} / \mathrm{Sn})$, and $\mathrm{Q}_{\mathrm{X}}$ is the average charge of $\mathrm{X}$ site $(\mathrm{Cl} / \mathrm{Br} / \mathrm{I})$.

\begin{tabular}{lccccccccc}
\hline \hline & \multicolumn{3}{c}{$\mathrm{C}^{-A B X} \mathrm{ABX}_{3}$} & \multicolumn{3}{c}{$\mathrm{T}-\mathrm{ABX}_{3}$} & \multicolumn{3}{c}{$\mathrm{O}-\mathrm{ABX}_{3}$} \\
& $\Delta \phi$ & $\mathrm{QB}_{\mathrm{B}}$ & $\mathrm{Q}_{\mathrm{x}}$ & $\Delta \phi$ & $\mathrm{QB}_{\mathrm{B}}$ & $\mathrm{Qx}$ & $\Delta \phi$ & $\mathrm{Q}_{\mathrm{B}}$ & $\mathrm{Qx}$ \\
\hline $\mathrm{CsPbCl}_{3}$ & 0.685 & 1.136 & -0.673 & 0.779 & 1.160 & -0.680 & 0.772 & 1.184 & -0.689 \\
$\mathrm{CsPbBr}_{3}$ & 0.352 & 0.884 & -0.589 & 0.847 & 1.057 & -0.642 & 0.875 & 1.072 & -0.646 \\
$\mathrm{CsPbI}_{3}$ & 0.778 & 0.799 & -0.550 & 0.843 & 0.874 & -0.575 & 0.894 & 0.895 & -0.579 \\
$\mathrm{CsSnCl}_{3}$ & 0.701 & 1.155 & -0.681 & 0.898 & 1.253 & -0.712 & 1.319 & 1.163 & -0.681 \\
$\mathrm{CsSnBr}_{3}$ & 0.666 & 1.025 & -0.634 & 1.047 & 1.114 & -0.659 & 1.220 & 1.072 & -0.646 \\
$\mathrm{CsSnI}_{3}$ & 1.010 & 0.843 & -0.562 & 1.041 & 0.897 & -0.579 & 1.079 & 0.912 & -0.583 \\
$\mathrm{RbPbCl}_{3}$ & -0.121 & 1.066 & -0.657 & 0.366 & 1.176 & -0.689 & 0.351 & 1.173 & -0.685 \\
$\mathrm{RbPbBr}_{3}$ & -0.042 & 0.880 & -0.591 & 0.439 & 1.015 & -0.631 & 0.514 & 1.055 & -0.641 \\
$\mathrm{RbPbI}_{3}$ & 0.064 & 0.702 & -0.527 & 0.533 & 0.863 & -0.576 & 0.608 & 0.886 & -0.578 \\
$\mathrm{RbSnCl}_{3}$ & 0.756 & 1.187 & -0.691 & 0.537 & 1.221 & -0.702 & 0.928 & 1.171 & -0.684 \\
$\mathrm{RbSnBr}_{3}$ & 0.739 & 1.111 & -0.662 & 0.566 & 1.130 & -0.668 & 0.901 & 1.049 & -0.638 \\
$\mathrm{RbSnI}_{3}$ & 0.693 & 0.827 & -0.562 & 0.573 & 0.913 & -0.589 & 0.827 & 0.871 & -0.573 \\
\hline \hline
\end{tabular}



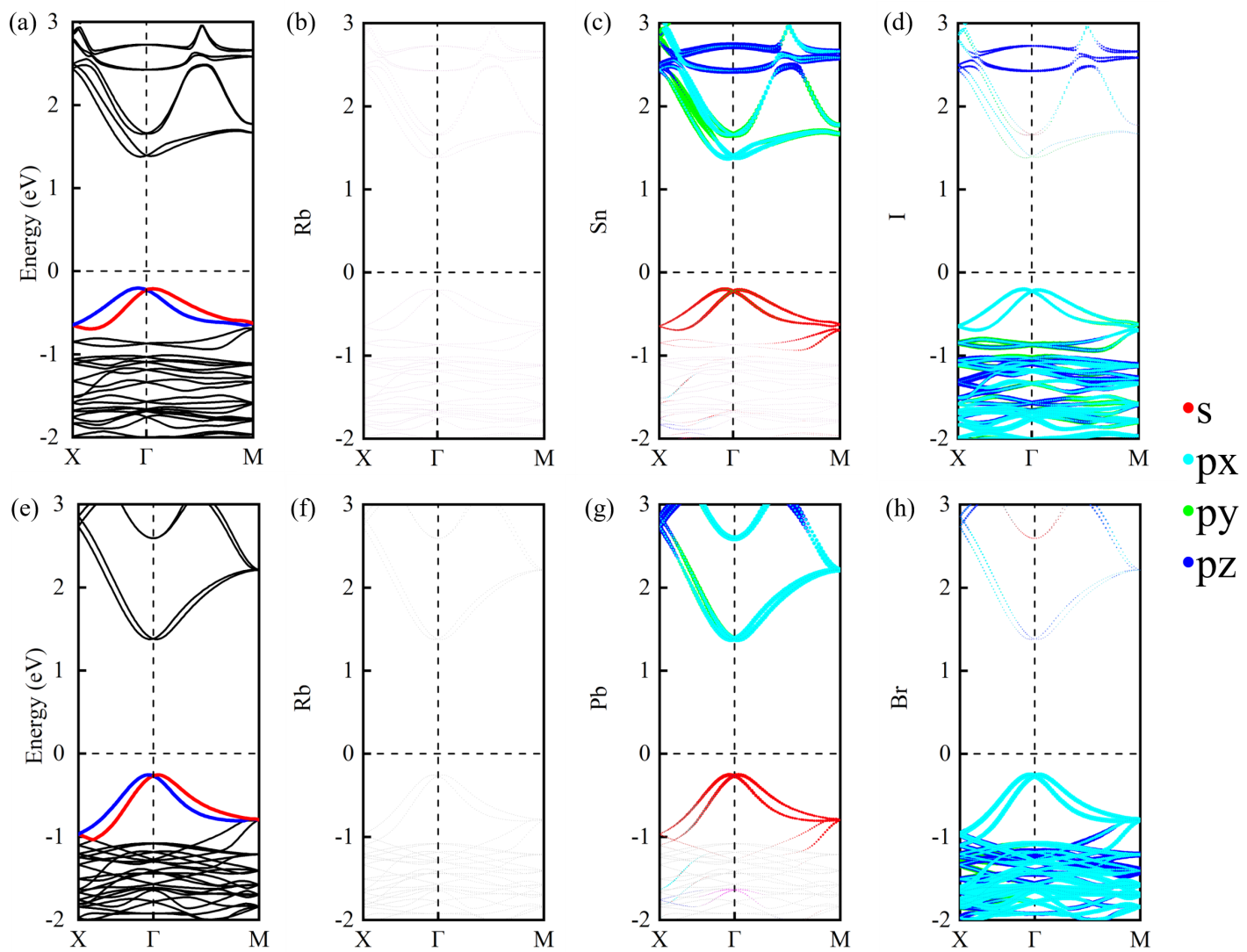

Fig. S4: The projected band structures of (a) O-RbSnI 3 with SOC for (b) Rb, (c) Sn and (d) I atoms. The projected band structures of (e) T-RbPbBr 3 with SOC for (f) Rb, (g) Pb and (h) Br atoms.
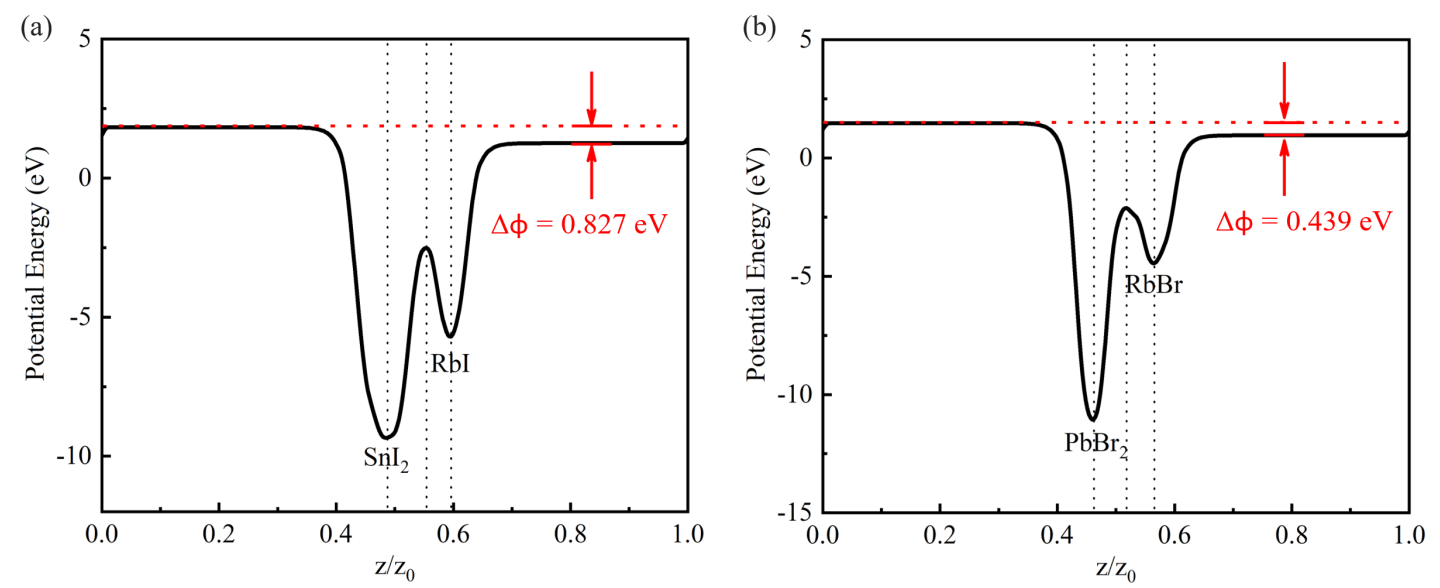

Fig. S5: The electrostatic potential energy of (a) $\mathrm{O}-\mathrm{RbSnI}_{3}$ and (b) $\mathrm{T}-\mathrm{RbPbBr}_{3} . \mathrm{Z}_{0}$ is the thickness of the unit cell. $\mathrm{Z}$ and $\mathrm{z} / \mathrm{z}_{0}$ denote the cartesian coordinates and fractional coordinates in the vertical direction, respectively. $\Delta \phi(\mathrm{eV})$ of $\mathrm{O}-\mathrm{RbSnI}_{3}\left(\mathrm{~T}-\mathrm{RbPbBr}_{3}\right)$ is the workfunction difference between $\mathrm{SnI}_{2}\left(\mathrm{PbBr}_{2}\right)$ and $\mathrm{RbI}(\mathrm{RbBr})$ side. 

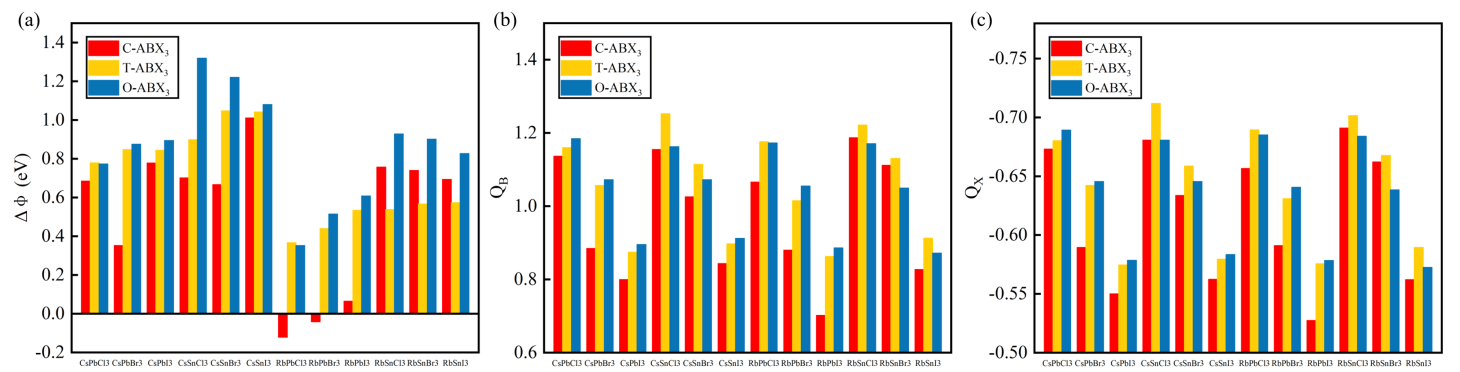

Fig. S6: (a) $\Delta \phi(e V)$ and (b) $\mathrm{Q}_{\mathrm{B}}$ and (c) $\mathrm{QX}_{\mathrm{X}}$ of $2 \mathrm{D} \mathrm{ABX}_{3}$ perovskites. 


\section{ELECTRONIC PROPERTIES OF CSPBI 3 3D BULK AND 2D NON-POLAR STRUCTURES}

The electronic properties of $2 \mathrm{D}$ and $3 \mathrm{D} \mathrm{CsPbI}_{3}$ are investigated using the first-principles density functional theory calculations. The 3D bulk has cubic, tetragonal and orthorhombic phases. When cutting through the (001) plane, two 2D non-polar perovskites appear, including CsI-terminated and $\mathrm{PbI}_{2}$-terminated slabs.
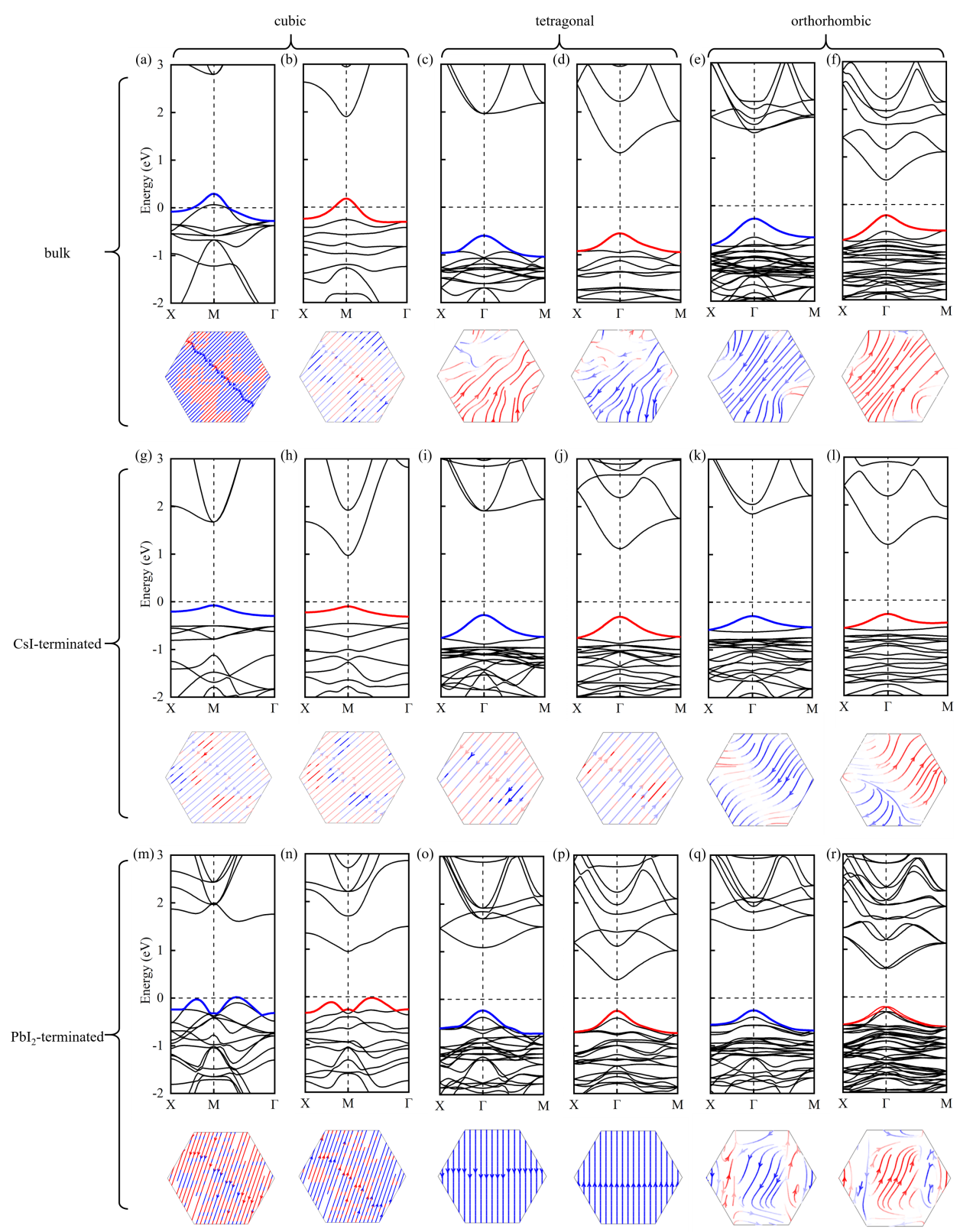

Fig. S7: Band structures of $\mathrm{CsPbI}_{3} 3 \mathrm{D}$ bulk and 2D non-polar structures without (left) and SOC (right). For each structure, the left band structure marks VBM in blue, and the right band structure marks VBM in red. The spin textures of the two topmost valence bands are shown at the bottom, where the left one is the spin texture of VBM and the right one is the spin texture of the valence band which is the closest to VBM. 


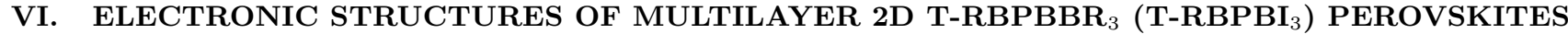

2D multilayer perovskites, such as $\mathrm{T}-\mathrm{RbPbBr}_{3}$ and $\mathrm{T}-\mathrm{RbPbI}_{3}$, still have Rashba effect around the $\Gamma$ point, but the Rashba constants are much smaller. Corresponding band structures and spin textures are depicted in Fig. S8.
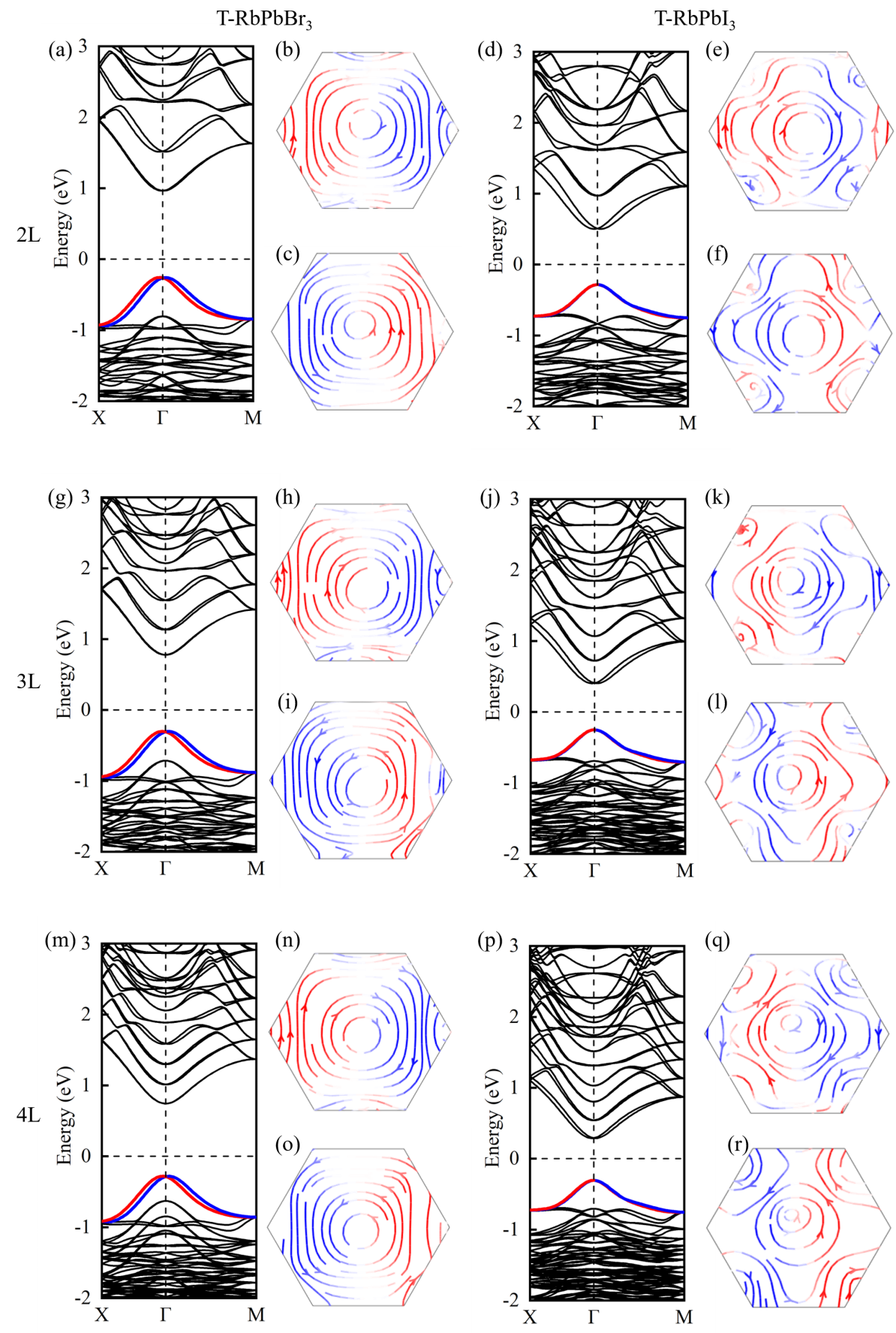

Fig. S8: Electronic structures of multilayer $2 \mathrm{D}$ polar perovskites ( $\mathrm{T}-\mathrm{RbPbBr}_{3}$ and $\left.\mathrm{T}-\mathrm{RbPbI}_{3}\right)$, including band structures with SOC and characteristic spin textures of upper and lower Rashba bands of VB. 


\section{STRUCTURE AND STABILITY}

2D T-ABX 3 and $\mathrm{O}-\mathrm{ABX}_{3}$ perovskites have Rashba effect and 2D C-ABX 3 perovskites lack Rashba effect. The lattice constants of $2 \mathrm{D}$ polar perovskites $\left(\mathrm{T}-\mathrm{ABX}_{3}\right.$ and $\left.\mathrm{O}-\mathrm{ABX} \mathrm{B}_{3}\right)$ are listed in Table $\mathrm{S} 3$.

Table S3: The lattice constants $(\AA)$ of $2 \mathrm{D}$ polar perovskites. Formation energy $\mathrm{E}_{\mathrm{f}}(\mathrm{eV}$ per f.u.) of 2D polar perovskites and corresponding $3 \mathrm{D}$ counterparts.

\begin{tabular}{ccccccccc}
\hline \hline \multirow{2}{*}{ structure } & \multicolumn{3}{c}{$\mathrm{T}-\mathrm{ABX}_{3}$} & \multicolumn{4}{c}{$\mathrm{O}-\mathrm{ABX}_{3}$} \\
& $\mathrm{a}$ & $\mathrm{b}$ & $\mathrm{E}_{f}(2 \mathrm{D})$ & $\mathrm{E}_{f}(3 \mathrm{D})$ & $\mathrm{a}$ & $\mathrm{b}$ & $\mathrm{E}_{f}(2 \mathrm{D})$ & $\mathrm{E}_{f}(3 \mathrm{D})$ \\
\hline $\mathrm{CsPbCl}_{3}$ & 8.107 & 8.107 & -2.13 & -1.43 & 8.175 & 8.021 & -0.48 & -0.61 \\
$\mathrm{CsPbBr}_{3}$ & 8.505 & 8.505 & -2.02 & -1.36 & 8.580 & 8.426 & -0.26 & -0.49 \\
$\mathrm{CsPbI}_{3}$ & 9.055 & 9.055 & -1.89 & -1.26 & 9.163 & 8.959 & 0.02 & -0.32 \\
$\mathrm{CsSnCl}_{3}$ & 7.959 & 7.959 & -2.28 & -1.40 & 8.513 & 8.127 & -0.88 & -0.67 \\
$\mathrm{CsSnBr}_{3}$ & 8.377 & 8.377 & -2.14 & -1.36 & 8.668 & 8.409 & -0.57 & -0.57 \\
$\mathrm{CsSnI}_{3}$ & 8.912 & 8.912 & -2.02 & -1.28 & 9.019 & 8.873 & -0.23 & -0.40 \\
$\mathrm{RbPbCl}_{3}$ & 8.106 & 8.106 & -1.94 & -1.33 & 8.134 & 7.969 & -0.37 & -0.57 \\
$\mathrm{RbPbBr}_{3}$ & 8.521 & 8.521 & -1.79 & -1.24 & 8.532 & 8.406 & -0.12 & -0.43 \\
$\mathrm{RbPbI}_{3}$ & 9.075 & 9.075 & -1.63 & -1.12 & 9.101 & 8.949 & 0.19 & -0.24 \\
$\mathrm{RbSnCl}_{3}$ & 7.962 & 7.962 & -2.14 & -1.30 & 8.383 & 7.993 & -0.81 & -0.61 \\
$\mathrm{RbSnBr}_{3}$ & 8.379 & 8.379 & -1.96 & -1.23 & 8.733 & 8.349 & -0.47 & -0.49 \\
$\mathrm{RbSnI}_{3}$ & 8.937 & 8.937 & -1.78 & -1.14 & 9.231 & 8.844 & -0.06 & -0.31 \\
\hline \hline
\end{tabular}

When studying a new material theoretically, it is essential to check whether it is stable. We prove the stability of 2D polar peroskites by investigating thier thermodynamical stability, dynamic stability and gas absorption.

First, the thermodynamical stability of $2 \mathrm{D}$ perovskites is examined by calculating the formation energy. The formation energies $\left(\mathrm{E}_{f}\right)$ of $2 \mathrm{D}$ and $3 \mathrm{D}$ perovskites $\left(\mathrm{ABX}_{3}\right)$ were calculated with respect to component layers $(\mathrm{AB}$ and $\mathrm{BX}_{2}$ ), which are defined as the following equation:

3D bulk:

$$
E_{f(3 D)}=E_{3 D}-E_{A X}-E_{B X_{2}}
$$

2D polar perovskites:

$$
E_{f(2 D)}=E_{2 D}-E_{A X}-E_{B X_{2}}
$$

Calculated formation energy of $\mathrm{CsPbBr}_{3}$ bulk with the orthorhombic phase from their binary components $(\mathrm{CsBr}$ $+\mathrm{PbBr}_{2} \rightarrow \mathrm{CsPbX}_{3}$ ) equals to $-0.49 \mathrm{eV}$ per f.u., which indicates that the ternary compound is stable. Although it is more negative than $-0.209 \mathrm{eV}$ per f.u. ${ }^{1}$ (PBE functional) and $-0.22 \mathrm{eV}$ per f.u. ${ }^{2}$ (PBEsol functional), the discrepancy does not change the comparison of the relative stability between bulk and 2D perovskites when the same functional is used for all perovskites. In this work, $\mathrm{T}-\mathrm{RbPbBr}_{3}$ and $\mathrm{T}-\mathrm{RbPbI}_{3}$ are good $2 \mathrm{D}$ materials for spin $\mathrm{FETs}$ due to their giant Rashba constant, strong response to the electric field and short channel lengths. As displayed in Table. S3, the calculated formation energies of $\mathrm{T}-\mathrm{RbPbBr}_{3}$ and $\mathrm{T}-\mathrm{RbPbI}_{3}$ are both negative, which indicate that the $2 \mathrm{D}$ structures are thermodynamically stable. In the meanwhile, the formation energies of 2D polar perovskites are much lower than their 3D counterparts, indicating the better thermodynamic stability of 2D polar perovskites.

Common 2D perovskites are non-polar structures. We then use $\mathrm{CsPbI}_{3}$ as an example and compare the formation energy of $2 \mathrm{D}$ polar $\mathrm{CsPbI}_{3}$ with $2 \mathrm{D}$ CsI-terminated and $\mathrm{PbI}_{2}$-terminated non-polar perovskites. For tetragonal phase, $2 \mathrm{D}$ polar $\mathrm{T}-\mathrm{CsPbI}_{3}$ are less stable than $2 \mathrm{D}$ non-polar perovskites. But $2 \mathrm{D}$ polar $\mathrm{CsPbI}_{3}$ are more stable than $3 \mathrm{D} \mathrm{CsPbI}_{3}$, which is pretty good. For orthorhombic phase, 2D AX-terminated $\mathrm{CsPbX}_{3}$ are thermodynamically more stable than the $\mathrm{BX}_{2}$-terminated $2 \mathrm{D}$ structures, consistent with previews research ${ }^{2}$. 2D polar O-CsPbI $\mathrm{O}_{3}$ are less stable than 2D CsI-terminated non-polar perovskites. But $2 \mathrm{D}$ polar $\mathrm{O}-\mathrm{CsPbI}_{3}$ are more stable than $2 \mathrm{D} \mathrm{PbI}_{2}$-terminated non-polar perovskites. 
(a)

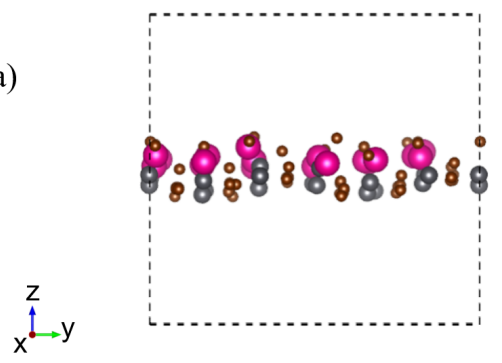

(b)

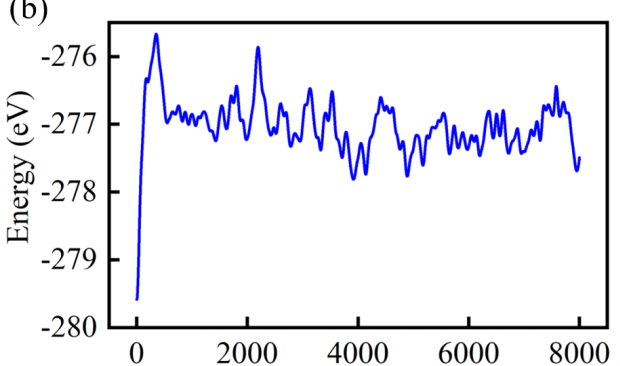

(c)

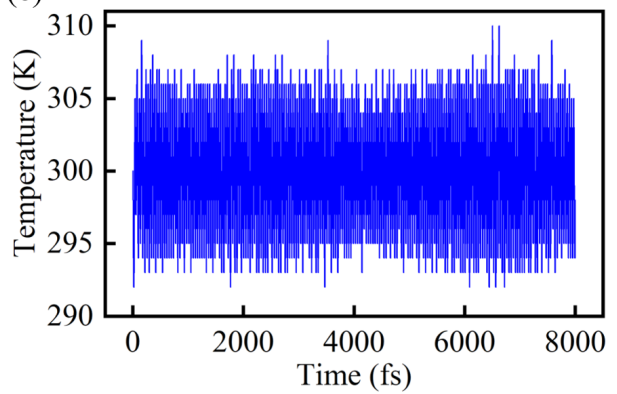

(d)

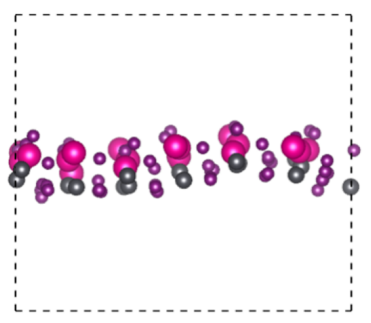

(e)

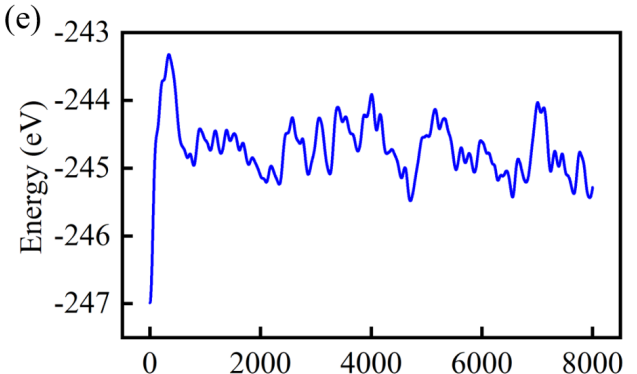

(f)

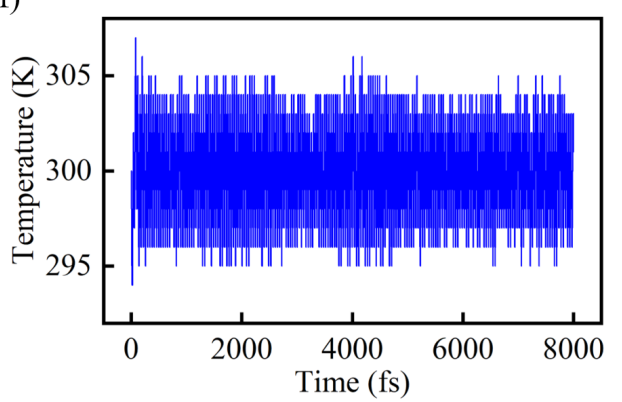

Fig. S9: Ab-initio molecular dynamics simulations of $\mathrm{T}-\mathrm{RbPbBr}_{3}$ and $\mathrm{T}-\mathrm{RbPbI}_{3}(\mathrm{~T}=300 \mathrm{~K})$. (a) The supercell structure of $\mathrm{T}-\mathrm{RbPbBr}_{3}$ after 8ps molecular dynamic calculations, and corresponding evolution of (b) total energy and (c) temperature. (d) The supercell structure of T-RbPbI3 after 8ps molecular dynamic calculations, and corresponding evolution of (e) total energy and (f) temperature.

Second, we perform ab initio DFT molecular dynamic simulations to further confirm the stability. we performed 8 ps molecular dynamics calculations of $\mathrm{T}-\mathrm{RbPbBr}_{3}$ and $\mathrm{T}-\mathrm{RbPbI}_{3}$. The results are shown in Fig. S9, indicating that the stability of these novel $2 \mathrm{D}$ polar perovskites at $300 \mathrm{~K}$.

Then, we further verify the dynamical stability of these 2D polar perovskites by calculating phonon spectrum. Taking $\mathrm{T}-\mathrm{RbPbI}_{3}$ as an example, unfortunately, $\mathrm{T}-\mathrm{RbPbI}_{3}$ has small imaginary frequencies of around $15 \mathrm{~cm}^{-1}$ as shown in Fig. R9. We attribute the imaginary frequency to the relatively small supercell. Our imaginary frequency is caused by the computational details. We have tried to optimized $3 \times 3 \times 1$ supercell with higher precision, and calculate phonon spectrum with more $\mathrm{K}$ points and higher cutoff. These adjustments of calculation parameters decrease the imaginary frequency, but these take more computing resources. Acoustic branches can be eliminated by increasing the size of the supercell, which need much more resources. In contrast, the previous studies report imaginary frequency for perovskites. For example, $\mathrm{LiOsO}_{3}$ with $\mathrm{R} \overline{3} \mathrm{c}$ room temperature phase have imaginary around $170 \mathrm{~cm}^{-13}$; The research of perovskites $\mathrm{Cs}_{2} \mathrm{AgInBr}_{(6-x)} \mathrm{Cl}_{x}$ also claimed that small imaginary frequency is acceptable errors in phonon calculations ${ }^{4}$. Therefore, combined with the molecular dynamics calculations and phonon spectrums, our 2D polar perovskites can be considered as stable structures.

We also use the h-BN monolayer to improve the stability of $2 \mathrm{D}$ polar perovskites. Here, we take $\mathrm{T}-\mathrm{RbPbI} 3$ as an example. The h-BN/T-RbPbI $3 /$ h-BN heterostructure with lattice mismatch less than $3 \%$ is shown in Fig. R10. The calculated Rashba constant of the h-BN/T-RbPbI $3 / \mathrm{h}-\mathrm{BN}$ heterostructure is $0.804 \mathrm{eV} \cdot \AA$, which is slightly above the value of $\mathrm{T}_{-} \mathrm{RbPbI}_{3}(0.708 \mathrm{eV} \cdot \AA)$. Therefore, we can conclude that h-BN can protect $2 \mathrm{D} \mathrm{T}-\mathrm{RbPbI} \mathrm{monolayer}_{3}$ and maintain its large Rashba constant. 


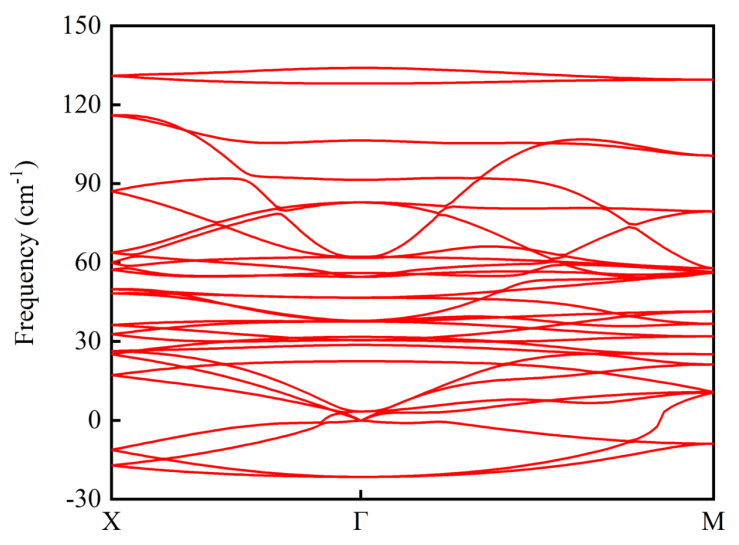

Fig. S10: Phonon spectra of $\mathrm{T}-\mathrm{RbPbI}_{3}$.

(a)

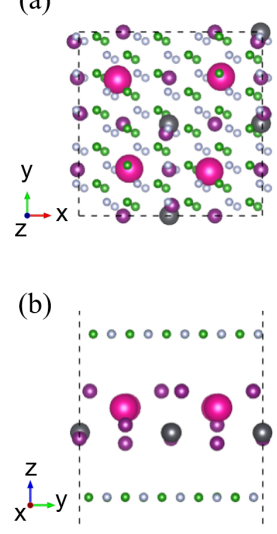

(c)

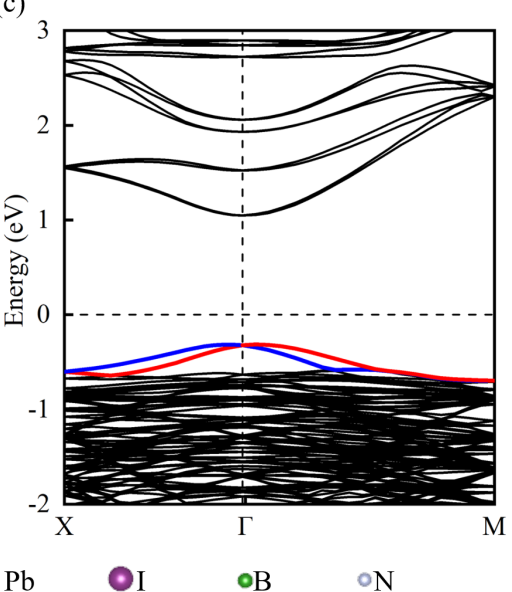

Fig. S11: (a-b) Atomic structure and (c) band structure of h-BN/T-RbPbI $3 / \mathrm{h}-\mathrm{BN}$.

Third, we check the stability of $2 \mathrm{D}$ polar perovskites $\mathrm{T}-\mathrm{RbPbBr}_{3}$ and $\mathrm{T}-\mathrm{RbPbI}_{3}$ in ambient conditions. The adsorption of gas molecules including $\mathrm{O}_{2}, \mathrm{H}_{2}, \mathrm{H}_{2} \mathrm{O}, \mathrm{CO}, \mathrm{CO}_{2}$ and $\mathrm{NH}_{3}$ are investigated. The binding energies and bond lengths of gas absorption are shown in Table $\mathrm{S} 4$. Binding energies, which are donated as $\mathrm{E}_{\mathrm{b}}$, were calculated by

$$
E_{b}=E_{\text {perovskite+gas }}-E_{\text {perovskite }}-E_{\text {gas }}
$$

Table S4: The binding energies $\mathrm{E}_{\mathrm{b}}(\mathrm{eV})$ and bond lengths $\mathrm{L}(\AA)$ of gas molecules on $\mathrm{T}-\mathrm{RbPbBr}_{3}$ and $\mathrm{T}-\mathrm{RbPbI}_{3}$.

\begin{tabular}{c|ccc|ccc}
\hline \hline \multirow{2}{*}{ Molecule } & \multicolumn{3}{|c|}{$\mathrm{T}-\mathrm{RbPbBr}_{3}$} & \multicolumn{3}{c}{$\mathrm{T}-\mathrm{RbPbI}_{3}$} \\
& $\mathrm{E}_{\mathrm{b}}$ & bond & $\mathrm{L}$ & $\mathrm{E}_{\mathrm{b}}$ & bond & $\mathrm{L}$ \\
\hline $\mathrm{O}_{2}$ & -0.03 & $\mathrm{Rb}-\mathrm{O}$ & 3.13 & -0.18 & $\mathrm{I}-\mathrm{O}$ & 4.57 \\
$\mathrm{H}_{2}$ & -0.15 & $\mathrm{Br}-\mathrm{H}$ & 3.36 & -0.18 & $\mathrm{I}-\mathrm{H}$ & 4.03 \\
$\mathrm{H}_{2} \mathrm{O}$ & -0.55 & $\mathrm{Rb}-\mathrm{O}$ & 2.85 & -0.59 & $\mathrm{Rb}-\mathrm{O}$ & 2.84 \\
$\mathrm{CO}$ & -0.25 & $\mathrm{Rb}-\mathrm{C}$ & 3.36 & -0.29 & $\mathrm{Rb}-\mathrm{C}$ & 3.38 \\
$\mathrm{CO}_{2}$ & -0.37 & $\mathrm{Rb}-\mathrm{O}$ & 3.17 & -0.37 & $\mathrm{~Pb}-\mathrm{O}$ & 3.31 \\
$\mathrm{NH}_{3}$ & -0.53 & $\mathrm{Rb}-\mathrm{N}$ & 3.04 & -0.59 & $\mathrm{~Pb}-\mathrm{N}$ & 2.76 \\
\hline \hline
\end{tabular}

The binding energies of $\mathrm{O}_{2}, \mathrm{H}_{2}$, and $\mathrm{CO}$ on $\mathrm{T}-\mathrm{RbPbBr}_{3}$ and $\mathrm{T}-\mathrm{RbPbI}_{3}$ are higher than -0.35 eV, corresponding bond lengths larger than $3.0 \AA$. The binding energies of $\mathrm{CO}_{2}$ and $\mathrm{NH}_{3}$ on $\mathrm{T}-\mathrm{RbPbBr}_{3}$ are smaller than -0.35 eV and 
their corresponding bond lengths larger than $3.0 \AA$, which means $\mathrm{T}-\mathrm{RbPbBr}_{3}$ can absorb $\mathrm{CO}_{3}$ and $\mathrm{NH}_{3}$ physically. Similarly, $\mathrm{T}-\mathrm{RbPbI}_{3}$ can absorb $\mathrm{CO}_{2}$ physically. But $\mathrm{T}-\mathrm{RbPbBr}_{3}$ can absorb $\mathrm{H}_{2} \mathrm{O}$ chemically, and $\mathrm{T}-\mathrm{RbPbI}_{3}$ can absorb $\mathrm{H}_{2} \mathrm{O}$ and $\mathrm{NH}_{3}$ chemically. Therefore, $\mathrm{T}-\mathrm{RbPbBr}_{3}$ and $\mathrm{T}-\mathrm{RbPbI}_{3}$ may degrade in ambient conditions. We can add h-BN monolayer to protect our $2 \mathrm{D}$ polar perovskites. Corresponding result has displayed in above discussion and Fig. S11. 
[1] Y.-Y. Zhang, S. Chen, P. Xu, H. Xiang, X.-G. Gong, A. Walsh, and S.-H. Wei, Chin. Phys. Lett. 35, 036104 (2018).

[2] Y. Yang, F. Gao, S. Gao, and S.-H. Wei, J. Mater. Chem. A 6, 14949 (2018).

[3] H. Sim and B. G. Kim, Phys. Rev. B 89, 201107 (2014).

[4] Y. Liang, J. Alloy. Compd. 862, 158575 (2021). 